Original Research Paper

\title{
Sperm DNA Fragmentation and Apoptosis Levels: A Comparison of the Swim up and the Density Gradient Centrifugation Methods for Sperm Preparation
}

\author{
${ }^{1,3}$ Silvia W. Lestari, ${ }^{2}$ Triyana Sari and ${ }^{1,3}$ Dwi A. Pujianto \\ ${ }^{I}$ Department of Medical Biology, Faculty of Medicine, Universitas Indonesia, Indonesia \\ ${ }^{2}$ Department of Biology, Faculty of Medicine, University of Tarumanegara, Indonesia \\ ${ }^{3}$ The Indonesian Reproductive Medicine Research and Training Center (Ina-Repromed), Indonesia
}

Article history

Received: 30-05-2016

Revised: 08-11-2016

Accepted: 11-11-2016

Corresponding Author:

Silvia W Lestari

Department of Medical

Biology, Faculty of Medicine

Universitas Indonesia,

Indonesia

Email: finallysilvia@gmail.com

\begin{abstract}
This study investigated the efficiency of two sperm preparation methods, namely, density gradient centrifugation and the swim-up method, in selecting spermatozoa based on the DNA fragmentation index and the apoptosis levels. Fifteen semen samples from infertile couples underwent sperm preparation in this study. The Makler ${ }^{\circledR}$ counting chamber and Eosin $\mathrm{Y}$ staining were used to analyse the sperm concentration, motility and viability. The sperm chromatin dispersion assay was used to determine the sperm DNA fragmentation index, while western blotting was used to determine the apoptosis levels. This study showed that Swim Up (SU) and Density Gradient Centrifugation (DGC) methods were able to select sperm with lower DFIs compared to sperm from whole semen. The SU method selected for sperm in both the "moderate" and "severe" DFI categories that have low DFIs better than the DGC method. Western blotting for the expression of caspase 3 in sperm showed a band of $\sim 35 \mathrm{kDa}$ and this band's intensity was lower in post-SU sperm compared to post-DGC sperm. Both methods selected for sperm with low caspase 3 expression, although the SU method selected for sperm with low activity of caspase 3 better than the DGC method. In addition to selecting for sperm with better progressive motility and viability, the SU and DGC methods also selected for sperm with low sperm DFIs and apoptosis levels.
\end{abstract}

Keywords: Swim-Up, Density Gradient Centrifugation, Sperm Dna Fragmentation, Apoptosis

\section{Introduction}

Intrauterine Insemination (IUI), an Assisted Reproductive Technique (ART), is a method for increasing the number of sperm that reach the fallopian tubes in order to increase the chances of fertilization. However, according to the American Pregnancy Association, the average success rate for IUI ranges from $10-20 \%$. Several prognostic factors for the success of IUI has been investigated, such as the woman's age, endometrial thickness, the number of follicles mature at the time of ovulation, the time and the frequency in which the insemination was performed, the percentage of sperm with abnormal morphology, the type and the percentage motility of sperm and the total number of sperm inseminated. However, there are couples who did not achieve any pregnancy even after several IUI attempts, despite no obvious abnormalities in both the men and the women (Muriel et al., 2006).

Studies have shown correlations between increased rates of miscarriage, pregnancy and ART failure with DNA fragmentation or low DNA integrity in sperm (Singh and Agarwal, 2011). Men with normal spermiogram examination results could be infertile and the problem may be associated with abnormalities in the sperm DNA (Jackson et al., 2010; Agarwal and Allamaneni, 2005; Shamsi et al., 2008). The incomplete formation and abnormal packaging of sperm chromatin during protamination can result in endogenous nicks on the DNA, which is characteristic of apoptosis and lead to the elimination of the defective germinal cells (Agarwal and Allamaneni, 2005). Therefore, sperm with normal DNA is required for fertilization and embryonic and fetal development. 
To increase the chances of pregnancy after IUI, ovulation can be synchronized either naturally or by stimulation with drugs and the sperm is prepared (washed) before injection into the uterus (Duran et al., 2002). Sperm preparation is performed to increase the fertilization capacity of the sperm (Chiamchanya et al., 2010). There are various methods of sperm preparation. However, two methods of preparation are currently the most frequently performed, namely the Swim-Up (SU) method and the Density-Gradient Centrifugation (DGC) method. Studies have investigated which of the two sperm preparation methods could select for better sperm quality, sperm concentration and motility.

Controversies regarding the advantages and disadvantages of the SU and the DGC methods have been reported by many studies. This study aimed to re-evaluate the efficiency of these methods in selecting for sperm before being used for IUI and based the evaluation on the sperm DNA fragmentation index and apoptosis level. This investigation included the measurement of the sperm DNA Fragmentation Index (DFI) by Sperm Chromatin Dispersion (SCD) assay and a confirmation test using caspase 3 activity detection by western blot analysis.

\section{Materials and Methods}

\section{Semen Collection and Analysis}

Semen samples were obtained from 15 men from infertile couples (normozoospermia) who underwent IUI. All subjects were Asian and partners of women who failed to conceive after 24 months of unprotected intercourse. The study was approved by the institutional ethical committee. Routine semen analysis was performed according to World Health Organization (WHO) guidelines (WHO, 2010). From each ejaculate, two aliquots were taken for SU and DGC preparation and another aliquot was taken for the SCD and the Western blot assay. The DFI samples were obtained as follow: in whole semen, there were 7 samples per each category, whereas in post-SU, there were 10 samples of good category, 4 samples of moderate category and 7 samples of severe category and in post-DGC, there were 8 samples of good category, 6 samples of moderate category and 7 samples of severe category.

\section{Swim-Up}

An aliquot of $0.5 \mathrm{~mL}$ whole semen was washed with $2 \mathrm{~mL}$ medium (Sperm preparation medium, Origio, Denmark) in a $15 \mathrm{~mL}$ Nunc conical tube (Thermo Scientific Nunc, New York, USA) and then centrifuged (Thermo Scientific Centrifuge, New York, USA) at $300 \times \mathrm{g}$ for $10 \mathrm{~min}$. The supernatant was discarded and the pellet was recovered and transferred to a tube containing $1 \mathrm{~mL}$ of sperm preparation medium. The tube was fixed at an angle of $45^{\circ}$ and incubated for $45 \mathrm{~min}$ at $37^{\circ} \mathrm{C}$. After incubation, the tube was fixed vertically and the upper phase was then gently aspirated with a Pasteur pipette (Falcon, New York, USA). An aliquot of the upper phase was further analysed for sperm quality.

\section{Density-Gradient Centrifugation}

A two-layer gradient was prepared with $2 \mathrm{~mL}$ each of 80 and $40 \%$ ready-to-use gradient media (Sil Select Plus, FertiPro NV, Belgium) in $15 \mathrm{~mL}$ Falcon tubes. Using a sterile pipette, $1 \mathrm{~mL}$ of a liquefied semen sample was placed on top of the upper gradient phase and centrifuged at $300 \times \mathrm{g}$ for $20 \mathrm{~min}$. Next, the supernatant was discarded and the pellet was suspended in $1 \mathrm{~mL}$ rinse medium and centrifuged again at $500 \times \mathrm{g}$ for $10 \mathrm{~min}$. The pellet was resuspended in $0.5 \mathrm{~mL}$ rinse medium. An aliquot of the resuspension was further analysed for sperm quality.

\section{SCD Assay}

The sperm was evaluated using the Spermfunc ${ }^{\circledR}$ DNAf kit (BRED Life Science Technology Inc., China). A microtube containing $0.1 \%$ low melting agarose was placed in a water bath set between $90-100^{\circ} \mathrm{C}$ for $5 \mathrm{~min}$ to melt the agarose. Once melted, the agarose was then transferred into a second water bath set at $37^{\circ} \mathrm{C}$ for $5 \mathrm{~min}$. Twenty-five microliters of the semen sample were added to the agarose and stirred until mixed. Twenty-five microliters of the sperm cell suspension in the agarose was then put into a $0.65 \%$ agarose-coated slide and covered with a $20 \times 20$ $\mathrm{mm}$ cover-slip. The slide was then refrigerated in a horizontal position at $4^{\circ} \mathrm{C}$ and the suspension was allowed to solidify for $5 \mathrm{~min}$. After the suspension had solidified, the coverslip was gently removed and the slide was incubated in a horizontal position with a denaturation solution containing $0.08 \mathrm{~N} \mathrm{HCl}$ at $22^{\circ} \mathrm{C}$ for $7 \mathrm{~min}$. The remaining denaturation solution was removed. The slide was then incubated with a lysis solution for $25 \mathrm{~min}$ at room temperature. The slide was then washed with $\mathrm{ddH}_{2} \mathrm{O}$ for $5 \mathrm{~min}$. A progressive dehydration series was performed by incubating the slide in increasing concentrations of ethanol (70, 90 and $100 \%$ ) for 2 min each. The slide was then air-dried and stained with Wright staining solution for $25 \mathrm{~min}$. The slide was then rinsed flowing water and dried. The staining intensity was observed under a light microscope. A total number of five hundred sperm cells per slide were classified according to the standard criteria for SCD assays for unfragmented DNA (big and medium halo) and fragmented DNA (small, no halo and degraded sperm cell) (Fernandez et al., 2005). The experiment was performed at least two times for each sample by one observer and the set of experiments were completed by at least two observers.

\section{Western Blot Analysis}

The total protein from sperm was extracted by solubilizing the sperms in Sodium Dodecyl Sulfate 
(SDS) buffer containing 10\% SDS, 10\% sucrose, 0.375 $\mathrm{M}$ Tris $\mathrm{HCl}(\mathrm{pH}$ 6.8) and a protease inhibitor cocktail supplement (Roche, Manneheim, Germany) for five min at $100^{\circ} \mathrm{C}$. Soluble protein was obtained by centrifugation at $9000 \times \mathrm{g}$ for $10 \mathrm{~min}$. Fifteen micrograms of protein were then separated by $10 \%$ SDS-PAGE and transferred to a Hybond-P PVDF membrane (Amersham, Bucinghamshire, UK) (Canale et al., 1994; Bjorndahl et al., 2005). The membranes were blocked using 5\% Bovine Serum Albumin in $1 \times$ TBST $(10 \mathrm{mM}$ Tris/ $\mathrm{HCl} 7.6,150$ $\mathrm{mM} \mathrm{NaCl}, 0.05 \%$ Tween 20) for $1 \mathrm{~h}$ at room temperature. The membranes were then incubated overnight at $4^{\circ} \mathrm{C}$ with caspase 3 rabbit polyclonal antibody (Cell Signaling Technology, Massachusetts, USA) used with a 1:1000 dilution. The antibody recognizes a protein with $\mathrm{MW}=35 \mathrm{kDa}$. The membranes were washed with $1 \times$ TBST three times for 5 min each and then incubated with a Horse-Radish Peroxidase (HRP)-conjugated goat anti-rabbit secondary antibody (Santa Cruz Biotechnology, Ltd., Texas, USA) used with a 1:1000 dilution for two hours at room temperature. The membranes were washed again with $1 \times$ TBST three times for $5 \mathrm{~min}$ each and HRP was detected using a Western blot chemiluminescence detection system (Amersham, Bucinghamshire, UK). The antigen-antibody reaction was detected using a Luminescent Image Analyzer (Imagequant $^{\mathrm{TM}}$ LAS 4000, GE Healthcare Life Sciences, Sweden). The intensity of the caspase 3 band was measured with ImageJ (NIH).

\section{Statistical Analysis}

Comparisons between the sperm parameter and DFI of sperm from whole semen and prepared sperm (post-SU or post-DGC), were performed using the Mann-Whitney test. The Mann-Whitney test was used too to compare the intensities of the caspase 3 bands in sperm from post-SU and post-DGC. The level of significance was set at $p<0.05$. Data were analyzed using SPSS (16th version).

\section{Results}

\section{Sperm Parameters in Whole Semen and Prepared Sperm}

The percentage of progressive motile sperm was significantly higher in prepared sperm $(\mathrm{p}=0.013$ at post-DGC and $p=0.001$ at post-SU) than in sperm from whole semen, while the number of progressive, motile sperm was higher in post-SU sperm than in post-DGC sperm. The percentage of viable sperm was also higher in post-DGC and post-SU samples than in whole semen, even though the results were not statistically significant (Table 1).

\section{Sperm DNA Fragmentation in Sperm from Whole} Semen and Prepared Sperm

Twenty one samples were used in the examination of sperm DNA fragmentation using the SCD assay. In the SCD assay, prepared semen samples predominantly contained sperm with large and medium halos (Fig. 1B and $1 \mathrm{C}$ ), whereas whole semen samples were dominated by sperm that were classified as small, no halos and degraded (Fig. 1A).

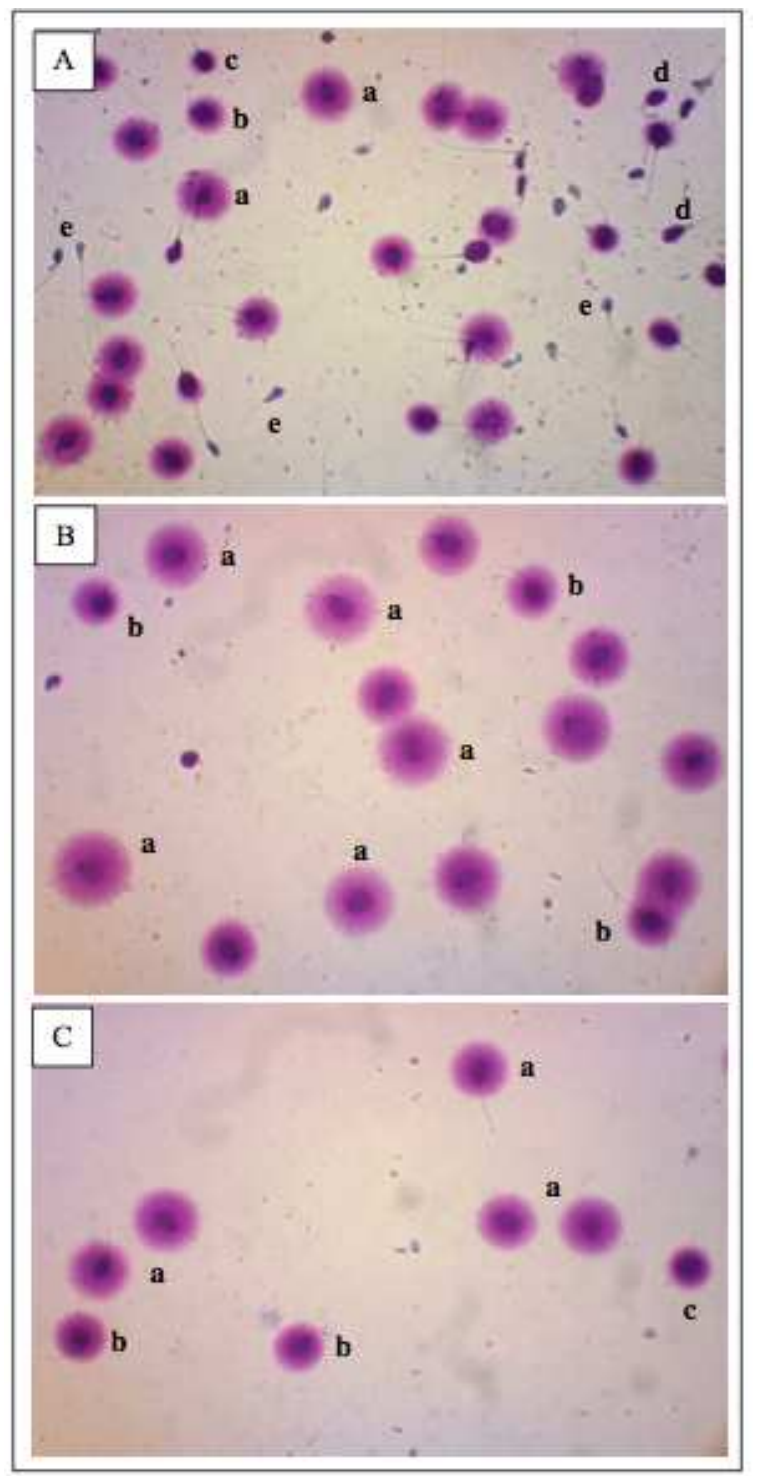

Fig. 1. The results of the sperm DNA chromatin (SCD) assay. (A) The result of the SCD assay in whole semen samples; (a) indicates a sperm with a large halo; (b) indicates a sperm with a medium halo; (c) indicates a sperm with a small halo; (d) indicates a sperm with no halo and (e) indicates a degraded sperm cell. (B) The result of the SCD assay in post-DGC samples. (C) The result of the SCD assay in post-SU samples. Both (B) and (C) showed more sperm with large and medium halos 


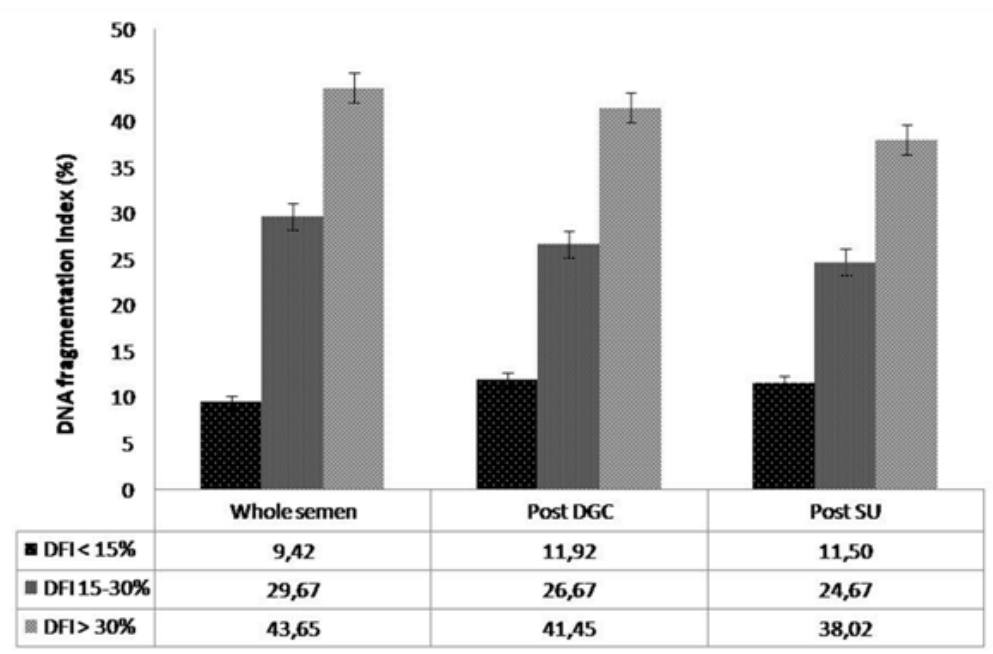

Fig. 2. The sperm DNA fragmentation index (DFI) of human sperm in whole semen and prepared semen. In the "good" category (black), the average DFIs of post-DGC and post-SU sperm were higher than the DFI of sperm from whole semen. In the "moderate" (dark grey) and "severe" categories (light grey), the average DFI of sperm of post-DGC and post-SU were lower than the DFIs of sperm whole semen

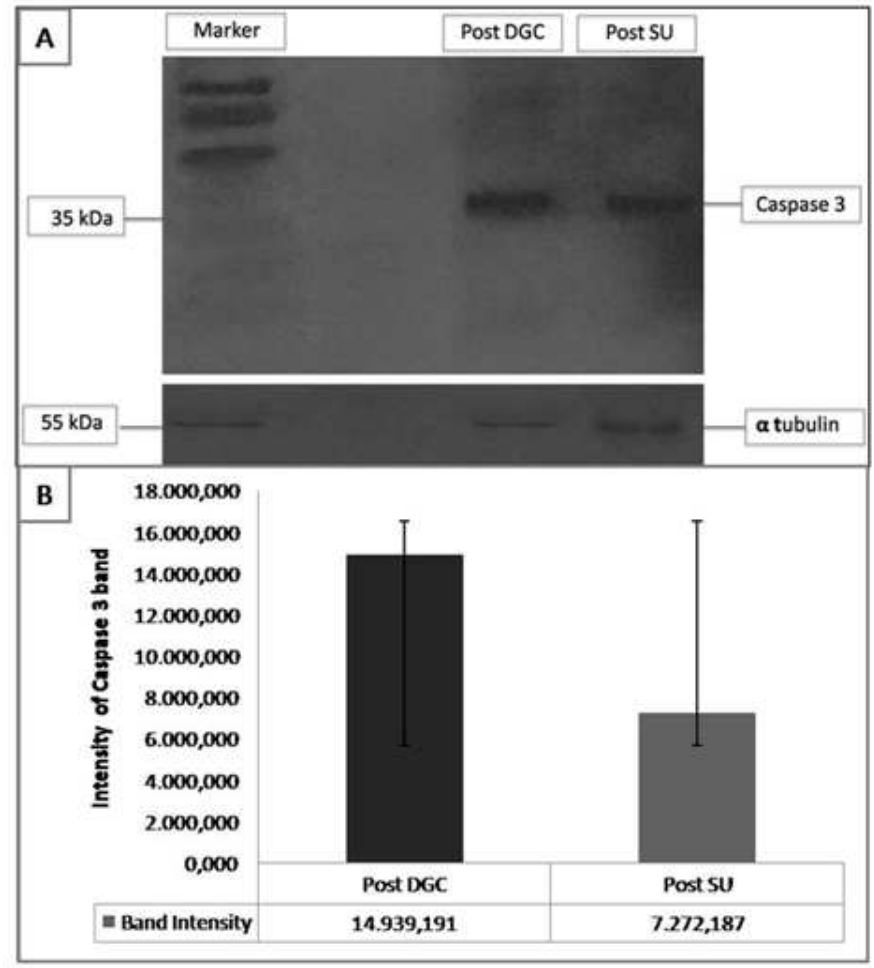

Fig. 3. Western blot analysis to detect caspase 3 expression in sperm from whole semen and prepared sperm. The result showed that the intensities of (A) the $\sim 35 \mathrm{kDa}$ band (B) were lower in post-SU compared to that of sperm from post-DGC. Tubulin was used as a housekeeping protein

Table 1. Conventional sperm parameters in whole and prepared sperm

\begin{tabular}{lllll}
\hline & Whole semen & Post DGC & Post US & p-value \\
\hline Percentage of motile sperm (\%) & $44.5 \pm 5.5$ & $65.33 \pm 4.2$ & $74.0 \pm 4.0$ & $0.013^{\mathrm{a}} ; 0.001^{\mathrm{b}}$ \\
Viable sperm (\%) & $63.2 \pm 4.04$ & $73.0 \pm 5.0$ & $73.17 \pm 5.05$ & $0.160^{\mathrm{a}} ; 0.164^{\mathrm{b}}$ \\
\hline
\end{tabular}

Note: Values are mean \pm SE; ' $a$ ' is the $p$ value from the comparison of sperm from whole semen and post-DGC semen; ' $b$ ' is the $p$ value from the comparison of sperm from whole semen and post-SU semen 
The DFI indicates the percentage of sperm with fragmented DNA in the sample. According to Mathwig et al. (2010) the DFIs can be classified into the following categories: (1) good (DFI 0-15\%), (2) moderate (DFI $>15-<30 \%$ ) and (3) severe (DFI $>30 \%$ ) (Mathwig et al., 2010). In this study, sperm that can be classified into the "good", "moderate" and "severe" DFI categories were observed. The result indicated that sperm from whole semen classified into the "good" category (black) had an average DFI of $9.42 \pm 0.56 \%$, whereas sperm from whole semen classified into the "moderate" category (dark grey) had an average DFI of $29.67 \pm 3.18 \%$ and sperm from whole semen classified into the "severe" (light grey) had an average DFI of $43.65 \pm 2.16 \%$. Meanwhile, post-DGC sperm classified into the "good" category had an average DFI of $11.92 \pm 1.24 \%$, whereas post-DGC sperm classified into the "moderate" category had an average DFI of $26.67 \pm 2.91 \%$ and sperm from post-DGC classified into the "severe" category (light grey) had an average DFI of $41.45 \pm 2.81 \%$ (Fig. 2). In addition, post-SU sperm classified into the "good" category had an average DFI of $11.5 \pm 1.11 \%$, whereas post-SU sperm classified into the "moderate" category had an average DFI of $24.67 \pm 5.69 \%$ and post-SU sperm classified into the "severe" category had an average DFI of $38.02 \pm 2.66 \%$. These results suggest that both post-DGC and post-SU sperm in the "moderate" and "severe" categories showed a lower DFI compared to whole semen $(\mathrm{p}<0.513$ and $\mathrm{p}<0.116)$, while post-DGC and post-SU sperm in the "good" category showed DFIs similar to that of sperm from whole semen $(\mathrm{p}<0.599)$, both were not statistically significant.

\section{Caspase 3 Expression in Sperm from Prepared Sperm}

Western blot analysis was performed to determine the expression of the caspase 3 protein in sperm. The result showed bands with molecular weights of $\sim 35$ $\mathrm{kDa}$ in all samples (Fig. 3A). A strong and bold band corresponding to caspase 3 expression was detected in both post-SU and post-DGC sperm, but post-SU sperm showed a smeared and weaker band (Fig. 3A). The result showed that the band intensities were lower in post-SU $(p<0.712)$ compared to that of sperm from post-DGC sperm $(p<0.712)$ (Fig. $3 B)$, both were not statistically significant.

\section{Discussion}

The selection process for the most efficient sperm is highly important for reproductive clinical practice. The efficiency of the sperm is measured as the percentage of motile sperm (WHO, 2010). This study showed that the percentage of motile sperm was significantly higher in prepared sperm (post-DGC and post-SU) than in sperm from whole semen and that the concentration of progressive motile sperm was higher post-SU than postDGC. The percentage of viable sperm was also higher in post-DGC and post-SU samples than in whole semen. However, it does not mean that the SU method is better than the DGC method because even the quality of the samples were the same (normozoospermia which has percentage of motile sperms $>32 \%$ ), but those samples have different percentage of motile sperms. The choice of the best technique for sperm preparation depends on the quality of the sample (Canale et al., 1994). If we have normozoospermia semen sample (i.e., normal concentration, motility and morphology), then we prefer the SU method. On the other hand, we prefer the DGC method for low quality semen samples (oligozoospermia, asthenozoospermia, teratozoospermia or combination). In addition, the SU method produces a higher level of non-sperm components (e.g., debris, bacteria) compared to the DGC method (Bjorndahl et al., 2005). Our study compared, for the first time, the sperm DNA fragmentation indices and the apoptotis levels of sperm prepared by the SU and the DGC methods. In addition to the routine semen parameters, this study also compared the DFIs of sperm prepared by either the SU or the DGC method to the DFIs of sperm from whole semen. The result showed that prepared sperm (either post-SU and post-DGC) had lower DFIs compared to sperm from whole semen. This study supports the finding by Jayaraman et al. (2012) that there was an elevation in DNA damage during the preparation of sperm. It assumed that the preparation methods during the sperm selection process affected the level of DNA integrity.

In the "moderate" and "severe" DFI category, the DFI of post-DGC sperm was higher than that of post-SU sperm. It means that the decrease in the DFI of post-SU sperm was greater compared to that of post-DGC sperm. Incubation at room temperature or $37^{\circ} \mathrm{C}$ after sperm isolation using the DGC method can reduce DNA fragmentation. In addition, repeated centrifugation during sperm preparation can induce iatrogenic DNA damage. This study supports the findings by Lampiao et al. (2010) that centrifugation for 10 and $30 \mathrm{~min}$ damaged both the motility and the viability of sperm and that $30 \mathrm{~min}$ centrifugation was more damaging than $10 \mathrm{~min}$. According to Marchesi et al. (2010) both the DGC and the SU methods can improve sperm DNA integrity, but the SU method improved the quality of DNA more than the DGC method. Xue et al. (2014) also stated that the SU method selected for sperm with reduced DNA fragmentation and vacuolization core better than the DGC method. In addition, this study also supports the finding by Jayaraman et al. (2012) that post-SU sperm obtained higher DNA integrity than post-DGC sperm. In addition, this study supports the findings by (Irvine et al., 2000; Zini and Sigman, 2009) that the two sperm preparation methods showed differences in the 
production of Reactive Oxygen Species (ROS) and sperm DNA damage.

Another finding of this study was the changes in caspase 3 expression, which represented the levels of apoptosis. In previous studies, caspase 3 expression was detected on the neck of the sperm and it was related to low sperm motility or decreased concentration, motility and abnormal morphology of the sperm (Almeida et al., 2005). However, it is not yet known whether ejaculated sperm are still able to initiate the apoptosis cascade or a marker of apoptosis in the sperm indicates a process of abortive apoptosis which was initiated before ejaculation (Lachnaud et al., 2004). In sperm, the activation of caspases is a sign of immaturity and is visible mostly in cases of male infertility. In addition, caspase activity in mature sperm can be observed after the induction of apoptosis in subphysiological conditions, such as during the cryopreservation process and thawing (Grunewald et al., 2009). Consequently, it can be presumed that post-ejaculation sperm has a poor ability to initiate apoptosis pathways and deaths primarily occur due to necrosis.

According to Grunewald et al. (2009) the analysis of caspase 3 activity in infertile men with oligoasthenoteratozoospermia and teratozoospermia indicated an increase in protease activity compared with the fertile men. This study supports the finding by Almeida et al. (2005) that there was a relationship between the expression of caspase 3 in sperm and decreases in both the morphology and the progressive motility of normal sperm. In some studies on sperm preparation, it was shown that the DGC method reduced the number of sperm that expressed caspase 3 in both fertile and infertile men. This reduction is related to the loss of immature sperm that have high caspase 3 expression. On the other hand, the SU method produced sperm with low caspase 3 expression in infertile men. This study showed that the intensity of the caspase 3 band in SU method was lower compared to the DGC method. Therefore, the low of caspase 3 expression in SU method was in line with the DFI level in post SU. In addition, the low expression of caspase 3 was in line with the percentage of motile sperms and the low of DFI after sperm preparation. These results correspond with the findings of (Grunewald et al., 2009).

\section{Conclusion}

This study is the first to compare the effects of the SU and the DGC methods on sperm DNA fragmentation and apoptosis levels. In terms of sperm preparation, the SU and the DGC methods were able to select for sperm with progressive motility and viability. In addition, the SU and DGC methods were able to select sperm with lower DFIs compared to sperm from whole semen. The SU method selected for sperm in both the "moderate" and "severe" DFI categories that have low DFIs better than the DGC method. Furthermore, both methods selected for sperm with low caspase 3 expression, although the SU method selected for sperm with low activity of caspase 3 better than the DGC method. In conclusion, the SU and the DGC methods can select for sperm with low DFIs, which can be confirmed by the low expression of caspase 3 .

\section{Acknowledgement}

The authors would like to express gratitude to the Indonesian Reproductive Medicine Research and Training Centre (Ina-Repromed) for supporting this study.

\section{Authors' Contributions}

Silvia W. Lestari: First author of the manuscript, experimental design, performance of the sperm preparation experiments, data collection and supervisory role of the project.

Triyana Sari: Experimental design, performance of the SCD assay, data collection, statistical analysis of data and manuscript review.

Dwi A. Pujianto: Experimental design, performance of the caspase 3 experiment and manuscript review.

\section{Ethics}

This article is original and contains unpublished material. The corresponding author confirms that all of the other authors have read and approved the manuscript and no ethical issues involved.

\section{References}

Agarwal, A. and S. Allamaneni, 2005. The effect of sperm DNA damage on assisted reproduction outcomes. A review. Minerva Ginecol., 56: 235-245.

PMID: 15258535

Almeida, C., M.F. Cardoso, M. Sousa, P. Viana and A. Gonçalves et al., 2005. Quantitative study of caspase-3 activity in semen and after swim-up preparation in relation to sperm quality. Hum. Reproduct., 20: 1307-1313.

DOI: $10.1093 /$ humrep/deh727

Bjorndahl, L., M. Mohammadieh, M. Pourian, I. Soderlund and U. Kvist, 2005. Contamination by seminal plasma factors during sperm selection. J. Androl., 2: 170-173. DOI: 10.1002/j.1939-4640.2005.tb01080.x

Canale, P.M., M. Giorgi, E. Gasperini, D. Pucci and M. Barletta et al., 1994. Inter and intra-individual variability of sperm morphology after selection with three different techniques: Layering, swimup from pellet and percoll. J. Endocrinol. Invest, 17: 729-732. DOI: $10.1007 /$ BF03347769 
Chiamchanya, C., N. Kaewnoonual, P. Visutakul, S. Manochantr and J. Chaiya, 2010. Comparative study of the effects of three semen preparation media on semen analysis, DNA damage and protamine deficiency and the correlation between DNA integrity and sperm parameters. Asian J. Androl., 12: 271-277. DOI: 10.1038/aja.2009.60

Duran, E.H., M. Morshedi, S. Taylor and S. Oehninger, 2002. Sperm DNA quality predicts intrauterine insemination outcome: A prospective cohort study. Hum. Reproduct., 17: 3122-3128.

DOI: $10.1093 /$ humrep/17.12.3122

Fernandez, J.L., L. Muriel, V. Goyanes, E. Segrelles and J. Gosálvez et al., 2005. Simple determination of human sperm DNA fragmentation with an improved sperm chromatin dispersion test. Fertility Sterility, 84: 833-842. DOI: 10.1016/j.fertnstert.2004.11.089

Grunewald, S., R. Sharma, U. Paasch, H.J. Glander and A. Agarwal, 2009. Impact of caspase activation in human spermatozoa. Microscopy Res. Technique, 72: 878-888. DOI: 10.1002/jemt.20732

Irvine, D.S., J.P. Twigg, K.L. Gordon, N. Fulton and P.A. Milne, 2000. DNA integrity in human spermatozoa: Relationships with semen quality. J. Androl., 21: 33-44. PMID: 10670517

Jackson, R.E., C.L. Bormann, P.A. Hassun, A.M. Rocha and E.L.A. Motta et al., 2010. Effects of semen storage and separation techniques on sperm DNA fragmentation. Fertility Sterility, 94: 2626-2630. DOI: $10.1016 /$ j.fertnstert.2010.04.049

Jayaraman, V., D. Upadhya, P.K. Narayan and S.K. Adiga, 2012. Sperm processing by swim-up and density gradient is effective in elimination of sperm with DNA damage. J. Assist. Reprod Genet, 29: 557-563. DOI: 10.1007/s10815-012-9742-x

Lachnaud, C., J. Tesarik, M.L. Cañadas and C. Mendoza, 2004. Apoptosis and necrosis in human ejaculated spermatozoa. Hum. Reproduct., 19: 607-610. DOI: 10.1093/humrep/deh130

Lampiao, F., H. Strijdom and S.S. Plessis, 2010. Effects of sperm processing techniques involving centrifugation on nitric oxide, reactive oxygen species generation and sperm function. Open Androl. J., 2: 1-5. DOI: $10.2174 / 1876827$ X01002010001
Marchesi, D.E., H. Biederman, S. Ferrara, A. Hershlag and H.L. Feng, 2010. The effect of semen processing on sperm DNA integrity: Comparison of two techniques using the novel Toluidine Blue Assay. Eur. J. Obstetr. Gynecol. Reproduct. Biol., 151: 176-180. DOI: 10.1016/j.ejogrb.2010.05.003

Mathwig, A., F. Topfer, L. Pfeiffer and B. Belitz, 2010. Detection of DNA damage in human spermatozoa with Halosperm Test. Halosperm Poster.

Muriel, L., M. Meseguer, J.L. Fernández, J. Alvarez and J. Remohí et al., 2006. Value of the sperm chromatin dispersion test in predicting pregnancy outcome in intrauterine insemination: A blind prospective study. Hum. Reproduct., 21: 738-744. DOI: 10.1093/humrep/dei403

Shamsi, M.B., R. Kumar and R. Dada, 2008. Evaluation of nuclear DNA damage in human spermatozoa in men opting for assisted reproduction. Ind. J. Med. Res., 127: 115-123. PMID: 18403788

Singh, A. and A. Agarwal, 2011. The role of sperm chromatin integrity and DNA damage on male infertility. Open Reproduct. Sci. J., 3: 65-71. DOI: $10.2174 / 1874255601103010065$

WHO, 2010. WHO laboratory manual for the examination and processing of human semen. 5th Edn., WHO Press, Geneve.

Xue, X., W.S. Wang, J.Z. Shi, S.L. Zhang and W.Q. Zhao et al., 2014. Efficacy of swim-up versus density gradient centrifugation in improving sperm deformity rate and DNA fragmentation index in semen samples from teratozoospermic patients. J. Assist. Reprod Genet, 31: 1161-1166. DOI: $10.1007 / \mathrm{s} 10815-014-0287-\mathrm{z}$

Zini, A. and M. Sigman, 2009. Are tests of sperm DNA damage clinically useful? Pros and cons. J. Androl., 30: 219 229. DOI: 10.2164/jandrol.108.006908 